\title{
Organisations sportives et recherche en management : entre acteurs publics et acteurs privés, des frontières qui bougent
}

Dans un modèle français de financement du sport qui laisse une place importante à la sphère publique, plutôt locale que nationale, le couple organisations privées (entreprises, associations) organisations publiques (État, collectivités) cohabite/collabore dans des modes de gestion variés et évolutifs depuis des décennies. Dans la continuité d'une doctrine issue de la Charte des sports de décembre 1940, elle-même modérée en 1943 par l'ordonnance d'Alger, texte renforcé par l'ordonnance du 28 août 1945, la première grande loi cadre d'octobre 1975 - dite Mazeaud - précisait dans son article 1: «Le développement de la pratique des activités physiques et sportives, élément fondamental de la culture, constitue une obligation nationale. Les personnes publiques en assument la charge avec le concours des personnes privées. » Cette double mention des «personnes publiques» qui prévalent et des personnes privées qui concourent illustre l'idée centrale d'une activité perçue à l'époque comme largement régalienne dans son essence mais - dans les faits - coproduite par des parties prenantes aussi bien publiques que privées. Durant cette période, le sport français - qui n'intègre pas encore les «activités physiques et sportives » qui apparaissent en 1984 (loi dite Avice) - est perçu dans une acception où les clubs associatifs et les fédérations qui les regroupent sont des acteurs centraux.

$\mathrm{Au}$ cours des dernières décennies, les modalités de la relation public/privé ont changé. Certains pans de l'économie du sport se sont développés dans une logique largement privée comme le secteur de la remise en forme ou le plein air de loisir qu'il soit de tourisme ou pour les résidents. D'autres s'inscrivent plus clairement dans une continuité historique autour des clubs associatifs et des fédérations sportives apparues au cours du $\mathrm{XX}^{\mathrm{e}}$ siècle. Toutefois, ces dernières formes d'activité ont, elles aussi, largement évolué dans un cadre global de professionnalisation et de commercialisation du secteur devenu très concurrentiel.

Ce numéro thématique propose huit contributions présentant des recherches questionnant l'intervention des acteurs publics et privés dans le cadre du sport. Ce faisant, les auteurs proposent une analyse de l'évolution des rapports public/privé, et parfois prédisent ou prescrivent les tendances à venir. Il s'agit d'un tour d'horizon - bien évidemment non exhaustif- de cas variés et divers.
Ainsi sont abordés l'organisation des évènements sportifs festifs ou compétitifs. La question du tourisme lié aux activités physiques est, elle aussi, développée et ce faisant les services sportifs de proximités sont analysés. Enfin, et très naturellement, le thème des équipements dédiés aux pratiques ou au spectacle sportif est analysé dans un secteur ou la sphère publique d'État ou locale intervient de manière importante.

Les objets d'étude concernent aussi bien le sport «qui se regarde » que celui «qui se pratique ». Dans tous les cas, les questionnements reprennent les jeux d'acteurs, autour de trois grands pôles. Le premier est celui des collectivités locales ou de l'État: la sphère publique est évidemment un des versants clé du secteur. Le deuxième pôle est celui du secteur privé, incarné par des structures juridiques constituées en sociétés privées de capitaux, souvent de type entreprise. Entre ces deux sphères se situe le monde associatif, très présent dans les activités physiques et sportives. Si la plupart des clubs sont constitués autour de ce statut, on observera que les fédérations sportives - tour à tour 《autorisées », «habilitées », 《agrées 》 ou 《soustutelle» en attendant le terme retenu dans le cadre de la réforme en cours - sont aussi des acteurs très présents. Elles ne peuvent être clairement positionnées comme appartenant à la sphère publique ou privée, d'où un statut parfois qualifié d'hybride.

La première partie de ce numéro propose une entrée par les fédérations. Ces entités ont vu leur organisation se transformer au cours des quatre dernières décennies. Que l'on envisage le cadre juridique de leur fonctionnement comme suivant ou précédant les changements organiques de ces organisations, les observateurs ne peuvent que constater un basculement majeur de leur logique de fonctionnement. La croissance des budgets, la professionnalisation de la pratique de haut-niveau, l'intérêt en termes de marketing que suscitent certaines compétitions ont amené ces entités a évolué fortement dans leur stratégie, dans un secteur qui plus est très concurrentiel. Toutefois les fédérations sportives restent toujours à ce jour des associations à but non lucratif disposant parfois de sociétés filiales commerciales. Ainsi elles se sont structurées et présentent aujourd'hui des organigrammes de PME - certes spécifiques - mais devant assurer leur pérennité, un suivi et un contrôle de leurs 
activités, sous l'œil des pouvoirs publics. Ainsi les fédérations, en tant qu'organisations sportives bénéficiant de prérogatives de puissance publique agissent sous le regard - le terme est volontairement large - d'un État qui n'oublie pas son rôle régalien, sans oublier son soutien financier.

Le premier article de ce numéro, proposé par TERRIEN, FEUILLET et BAYLE, envisage une analyse de la performance économique et financière de 76 fédérations françaises sur la période 2012-2017. L'outil statistique principal est celui d'une analyse factorielle. Ces analyses permettent d'identifier les vecteurs de vulnérabilités financières, mais aussi de proposer un cadre de lecture systémique de la performance économique et financière des fédérations sportives. Les auteurs mettent en avant trois dimensions majeures évaluées chacune par un score unique: dépendance en ressources publiques; budget financier et attraction de ressources financières; investissement dans les services. Le lecteur notera l'importance de la stabilité du lien quant au financement public, même s'il n'est pas unique dans les facteurs identifiés.

La deuxième contribution proposée par BAYLE, concentre son propos sur la fédération française de tennis (FFT), deuxième organisation sportive en termes de licences délivrées, propriétaire de son siège - le Stade de Roland Garros - et par ailleurs de tournois professionnels du circuit de la Fédération International de Tennis (ITF) ou de l'Association des joueurs professionnels (ATP). Dans une analyse qualitative longitudinale, l'auteur présente une lecture permettant de comprendre les spécificités de la FFT. L'approche historique de 1970 à nos jours permet de comprendre comment les relations du public et du privé ont entraîné une gouvernance de compromis pour ancrer une stratégie de développement ayant pour objet d'optimiser et de légitimer les soutiens publics. Dans ce modèle, la dimension économique de la structure s'est construite autour d'un évènement: le tournoi du Grand Chelem de Roland Garros. L'événement constitue un fil directeur fort dans une vision historique de la FFT. L'article décrit ainsi les tiraillements de cette fédération importante entre relations avec l'État (et la ville de Paris) et pressions du contexte social dans lequel elle évolue. Il s'agit alors de discuter de ce que révèlent les stratégies d'autonomisation mais aussi d'acceptation et de gestion des dépendances par rapport à la sphère publique.

L'article suivant rédigé par MARSAC se penche lui aussi sur une fédération, celle de Canoë-kayak (FFCK). Cette organisation est comme la FFT confronté à une logique de développement où les pratiques compétitives, largement coordonnées par les clubs sportifs, se trouvent complétées par des activités de loisir, essentiellement touristiques. Ce questionnement se retrouve dans de nombreuses autres disciplines de plein air : ski, équitation, voile, etc. L'auteur s'appuie sur deux sources congruentes. La première est un sondage IFOP et la seconde un corpus de vingt entretiens auprès de cadres techniques fédéraux et d'élus de la FFCK. L'approche identifie la manière, pour ne pas dire la stratégie, dont la fédération va réagir à l'émergence de pratiques nouvelles, proposées par des entreprises privées mais aussi des fédérations affinitaires. Après l'identification de quatre grandes périodes, l'article développe l'avenir des possibles en termes de positionnement de la FFCK quant aux pratiques de tourisme.

La quatrième contribution se penche lui aussi sur les fédérations sportives mais sous un angle différent. À travers le cas du football, RUPPE et DERMIT analysent les formes de régulation disciplinaire de l'éthique que les fédérations et leurs ligues professionnelles ont mis en place sous la pression des pouvoirs publics, via deux textes législatifs votés en 2012 et 2017. L'article envisage la manière donc les organisations sportives ont répondu à ces quasi injonctions de leur tutelle publique à réguler les incivilités telles que dopage, violence, corruption. Dans le cadre général de l'éthique sportive, les auteurs se focalisent ici sur la manière dont les comportements des joueurs - qui sont aussi des professionnels au travail dans ce cas - sont régulés par les instances fédérales. Le corpus documentaire sur lequel s'appuie la recherche est très important et essentiellement constitué autour du football. Il permet notamment de mieux cerner l'effectivité des sanctions prononcées par rapport à celles possibles. L'analyse statistique est complétée par une vision en recul des chiffres, démarche salutaire en termes de recherche.

La deuxième partie de ce numéro se focalise sur des acteurs privés dans leurs relations avec la sphère publique. Les quatre contributions suivantes concernent ainsi dans leur orientation principale les clubs sportifs professionnels. Qu'il s'agisse de football ou de sports en salle, ces organisations évoluent dans un écosystème mêlant les parties prenantes publiques et privées.

Ainsi dans le cinquième article, FRANÇOIS, BOUCHER et DERMIT se penchent sur l'engagement sociétal des clubs de football professionnel-au-delà de leur objet social initial. La notion de responsabilité sociale n'est pas propre aux seules entreprises privées de spectacle sportif. Toutefois, le secteur sous la pression de son environnement politique, s'est engagé dans cette direction, l'ensemble des équipes de Ligue 1 disposant d'au moins une structure ayant pour objet une fonction sociale de type fondation/fonds de dotations ou association à objet social. Comme le notent les auteurs, ce mouvement puissant, ponctué par un foisonnement des initiatives, relève d'une pression très ancienne des pouvoirs publics. La contrepartie aux financements publics (subventions directes et indirectes et/ou construction d'enceintes dédiées) se trouve dans des missions d'intérêt général assurées par les clubs professionnels. À travers huit entretiens et un corpus documentaire large (statuts et rapports d'activité), l'article questionne l'efficacité des actions et la restauration éventuelle de la légitimité des clubs en tant qu'acteurs sociaux sur leurs territoires.

La contribution suivante proposée par MOULARD et DERMIT, concerne la question des partenariats publicprivé (PPP) dans le cas du programme de construction/ rénovation des stades de football en France à l'occasion de 
l'Euro 2016. Les auteurs analysent les effets de ce programme important largement financés par le contribuable principalement local mais aussi national. La méthode s'appuie sur huit entretiens réalisés auprès de cadres des clubs et d'universitaires experts des enceintes sportives. De plus, le corpus documentaire comprend une masse importante de données financières qui permettent la construction d'indicateurs synthétiques des plus éclairants sur la réalité des effets obtenus par ces nouvelles enceintes. Outre le diagnostic posé, le texte propose dans sa dernière partie quelques pistes pour l'avenir des PPP constitués dans le cadre de la gestion des enceintes sportives.

Le $7^{\mathrm{e}}$ article quitte le monde du football pour se pencher sur les sports d'arénas: basket, handball et volleyball. Ces sports sont «traditionnellement» largement subventionnés par les collectivités locales : ville, intercommunalité, département et région. Comme le notent TERRIEN, VALIN et JUGE, les volumes financiers sont sensiblement moins importants que dans le football ou même que dans le rugby. De ce fait, le ticket d'entrée financier est réduit, permettant à des territoires d'importance moindre d'exister sur la scène du sport professionnel pour un coût public tenable pour des collectivités disposant de moindres moyens. La recherche réalise un focus sur la capacité des clubs à mobiliser financièrement leur zone de chalandise à travers leur expertise dans le domaine commercial: se vendre, y compris auprès des élus! À travers un corpus de données secondaires croisé par trois entretiens réalisés auprès de directeurs administratifs et financiers dans chacun des trois sports, les auteurs proposent une lecture très originale des types de modèles auxquels répondent les clubs de sport indoor et notamment de leur dépendance/indépendance à l'égard de la sphère publique locale.
La contribution qui ponctue ce numéro change de focale. L'article proposé par RAHIB et BODIN envisage en effet la question de la sécurisation des manifestations et des enceintes sportives à travers le prisme d'une coopération « légale ou imposée » entre l'État dans son rôle régalien et les entreprises de spectacle sportif dans leur logique commerciale. Ainsi, les auteurs identifient une transformation au cours du temps vers une collaboration qu'ils qualifient de «stratégique », tant pour les organisateurs cette dimension doit être prise en compte pour perdurer et se développer. L'analyse longitudinale de l'évolution législative trouve un terrain d'observation privilégié à travers un cas emblématique des questions de sécurité réelle et perçue des enceintes sportives : le Paris Saint-Germain et son Parc des Princes. L'intérêt de la démonstration est une lecture renouvelée d'une évolution réglementaire visant des objectifs différents entre les acteurs mais - par nécessité - devant trouver un modus operandi entre sphère publique et sphère privée.

Ces huit articles illustrent la variété et le dynamisme de la recherche autour des rapports entre acteurs publics et privés autour d'un sujet plus général des fondements de l'action collective et de la régulation du système sportif. S'y mêlent des enjeux sociaux - donc politiques - et financiers donc économiques. Ainsi, se voit se confirmer l'intérêt d'une approche de type «Economie politique» du sport, proposée par Wladimir Andreff depuis les années 1990.

Les éditeurs souhaitent remercier les reviewers de ce numéro pour leur lecture attentive et experte ainsi que les éditeurs de la revue aussi bien son rédacteur en chef que les responsables du domaine SHS de la publication pour leur suivi constant, dans une période troublée du fait de la crise sanitaire.

Christophe DURAND Jeanne-Maud JARTHON

Citation de l'article : Durand C \& Jarthon J-M (2021) Organisations sportives et recherche en management: entre acteurs publics et acteurs privés, des frontières qui bougent. Mov Sport Sci/Sci Mot, 111, 1-3 